\title{
Metodología y herramienta VERDE para la evaluación de la sostenibilidad en edificios
}

\author{
$V E R D E$, a methodology and tool for a sustainable building \\ assessment
}

M. $\operatorname{Macías}^{(*)}$, L. García Navarro ${ }^{(* *)}$

\section{RESUMEN}

\begin{abstract}
La edificación sostenible ha crecido a partir del movimiento hacia la edificación verde y bajo el movimiento más amplio de desarrollo sostenible. El movimiento verde se desarrolla en los años 70 con especial énfasis en la conservación de la energía y la eficiencia energética. En los 80 crece la preocupación acerca del impacto que produce la operación del edificio y la fabricación de los materiales de construcción sobre el medioambiente natural. Durante esta misma década los problemas de la pobre calidad del aire interior y la inadecuada ventilación en edificios herméticos (síndrome del edificio enfermo) constituyen una preocupación creciente en los ciudadanos. Gradualmente ha habido un incremento en el consenso en relación con el tipo impactos que deben incluirse en un modelo de evaluación verde.
\end{abstract}

Desde el año 2000, el número de métodos para mundo se ha multiplicado considerablemente. BREEAM (BRE Environmental Assessment Method) fue el primer sistema (aparecido en 1990) que ofreció un método de etiquetado de edificios aunque LEED (Leadership in Energy and Environmental Design) es el de mayor implantación en el mercado de grandes edificios. Actualmente existe un gran número de modelos, muchos de ellos basados en la metodología desarrollada por el grupo GBC (Green Building Challenge), actualmente iiSBE (International Initiative for a Sustainable Built Environment). En este trabajo se presenta una nueva metodología y un modelo de evaluación de la sostenibilidad de edificios denominado VERDE, basado en la cuantificación de la reducción de impactos.

Palabras clave: edificación sostenible, criterio, categorías de impacto, indicadores de sostenibilidad, evaluación ambiental. la evaluación medioambiental de edificios en el

\begin{abstract}
SUMMARY
Sustainable Building has grown out of a synthesis of the environmentally-oriented Green Building movement and the broader SD movement. The green movement itself grew out of 1970's concerns about Energy Conservation, which subsequently morphed into Energy Efficiency, as fuel prices declined during the 1980's. During the 1980's, energy issues receded somewhat into the background of general discussion, but concerns arose about the impact that building operations and the production of their constituent materials have on the natural environment. During the same decade, the issue of poor indoor air quality and inadequate ventilation in sealed buildings ("sick building syndrome") became a very public concern. A consensus has gradually evolved concerning the type of issues that should be included within a green type of analysis.
\end{abstract}

Since 2000 the number of environmental assessment methodologies around the world has been increasing rapidly. BREEAM (BRE Environmental Assessment Method) was the first system (launched in 1990), to offer an environmental label for buildings. Nevertheless, LEED (Leadership in Energy and Environmental Design) has been an incontrovertible success as a tool to promote market transformation. There are a number of different schemes around the world, most of which have been based on GBC and iiSBE (International Initiative for a Sustainable Built Environment). A new assessment method based on impact reduction calculation has been developed. The tool call VERDE is applicable to commercial complexes and residential developments. The methodology are presented and discussed in the paper.

113-91

Keywords: sustainable buildings, impact categories, criteria, sustainable indicators, environmental assessment.

\footnotetext{
(") Prof. Titular de la UPM, Grupo de Investigación ABIO-UPM, Dpto. de Ordenación del Territorio, Urbanismo y Medio Ambiente, Universidad Politécnica, Madrid (España)

${ }^{(*)}$ Prof. Titular de la UPM, Grupo de Investigación SCI-UPM, Dpto. de Construcción y Vías Rurales, Universidad Politécnica de Madrid (España)

Persona de contacto/Corresponding author: justo.gnavarro@upm.es (J. García Navarro)
}

Informes de la Construcción Vol. 62, 517, 87-100, enero-marzo 2010 ISSN: 0020-0883 elSSN: 1988-3234 doi: 10.3989/ic.08.056 


\section{ANÁLISIS DE LA METODOLOGÍA ACTUAL Y VENTAJAS DE LA PROPUESTA}

Los métodos de evaluación se estructuran en tres grandes grupos:

Aquellos basados en la valoración de actuaciones, establecidas en créditos a los que se asocia un número de puntos en función de la importancia en los impactos asociados al crédito. En este grupo se encuentran los modelos LEED V3 (USGBC) ${ }^{1}$ y BREEAM (BRE-GB) ${ }^{2}$.

El método de evaluación de CASBEE (Japón) ${ }^{3}$ se basa en el concepto de ecoeficiencia, definido como "valor de productos y servicios por unidad de cargas medioambientales". La Eficiencia Medioambiental del Edificio que usa CASBEE como indicador se define como una relación entre las categorías de "Rendimiento y Calidad Medioambiental del Edificio" y las "Cargas Medioambientales asociadas".

La plataforma de investigación $\mathrm{GBC}^{4}$ ha desarrollado la herramienta GBTool, que permitió que muchos grupos nacionales particularizaran la herramienta a las condiciones nacionales. GBTool utiliza una estructura jerárquica de árbol constituida por áreas, categorías y criterios.

España ha tenido una larga historia de participación en GBC, y eso se refleja en los condicionantes que la GBTool ha tenido en el desarrollo de la herramienta de evaluación española VERDE ${ }^{5}$.

En la última Conferencia Mundial Sustainable Building 2008 (SB08) ${ }^{6}$, celebrada en Melbourne (Australia), fue presentada una nueva propuesta basada en la evaluación de impactos y en los últimos proyectos normativos en desarrollo, tanto en ámbitos internacionales mundiales (ISO/TC 59/SC 17 Sustainability in building construction $)^{7}$, como a nivel europeo (CEN/TC 350 Sustainability of construction work $)^{8}$.

La herramienta de evaluación VERDE recoge los planteamientos de dichas propuestas normativas y evalúa la reducción de los impactos del edificio y su emplazamiento por la implementación de medidas, tanto en estrategias de diseño como en factores de rendimiento, agrupadas en una lista de criterios.

El procedimiento utiliza un método prestacional similar al método de evaluación energética de edificios.

Generalmente las metodologías de ponderación de pesos están basadas en un proceso de consenso entre expertos. Con la nueva orientación de la herramienta VERDE se ha intentado reducir el grado de subjetividad, introduciendo un sistema de valoración de la reducción de impactos con los sistemas de cálculo actuales. A cada criterio se le asocia un peso por cada uno de los impactos asociados, que refleja la extensión, la duración y la intensidad para realizar una evaluación relativa. Para la evaluación de los impactos absolutos, el peso dado a las categorías de impacto está basado en la política medioambiental española y en los datos relativos a los indicadores de sostenibilidad, como se refleja en el informe del Observatorio de Sostenibilidad Español ${ }^{9}$.

\section{DESCRIPCIÓN DE LA METODOLOGÍA VERDE}

La mayoría de los sistemas de evaluación (1) mezclan dos tipos diferentes de información: las medidas incorporadas al proyecto y los impactos asociados a las medidas. Ello conduce a estos sistemas a un intento de resolver dos funciones en una: guiar a los promotores y proyectistas en el intento de diseñar edificios de alto rendimiento (Guía de diseño) y evaluar el rendimiento del edificio de la forma más objetiva posible (Herramienta de evaluación).

El modelo propuesto trata de proporcionar al sector de la edificación una metodología y herramienta actualizada y homologable internacionalmente que permita, de forma objetiva, la evaluación de la sostenibilidad de los edificios, difundiendo a su vez los principios y buenas prácticas para su diseño y construcción.

La metodología VERDE está basada en una aproximación al análisis de ciclo de vida en cada fase (2-3) y consiste en evaluar la reducción de los impactos del edificio y su emplazamiento por la implementación de medidas, tanto en estrategias de diseño como en factores de rendimiento, agrupadas estas medidas en una lista de criterios de sostenibilidad.

Los criterios son entidades que permiten caracterizar el edificio a través de aspectos específicos (consumo de la energía primaria, emisiones de $\mathrm{CO}_{2}$, consumo de agua potable, etc.). Para hacer operativa la evaluación de cada criterio, es necesario asociar cada criterio con uno o más impactos y el indicador que suministra un valor numérico y su unidad de medida $\left(\mathrm{kWh} / \mathrm{m}^{2}\right.$ año, $\mathrm{kg} \mathrm{CO}$ equiv./m² año, l/persona día).

Los criterios a evaluar se estructuran en dos grandes grupos: los relacionados con la parcela (A) y los asociados al edificio agrupados en distintas categorías (B-F). 


\section{A Parcela y emplazamiento}

1. Estrategias para la clasificación y el reciclaje de residuos.

2. Uso de plantas autóctonas.

3. Uso de árboles para crear áreas de sombra.

4. Efecto isla de calor a la altura del suelo.

5. Efecto isla de calor a la altura de la cubierta.

6. Contaminación lumínica.

\section{B Energía y atmósfera}

7. Uso de energía no renovable incorporada en los materiales de construcción.

8. Energía no renovable en el transporte de los materiales de construcción.

9. Consumo de energía no renovable durante la fase de uso. Demanda y eficiencia de los sistemas.

10. Demanda de energía eléctrica en la fase de uso.

11. Producción de energía renovable en la parcela.

12. Emisiones de sustancias foto-oxidantes en procesos de combustión.

13. Emisiones de sustancias que reducen el ozono estratosférico.

\section{Recursos naturales}

14. Consumo de agua potable.

15. Retención de aguas de lluvia para su reutilización.

16. Reutilización de aguas grises.

17. Impacto de los materiales de construcción. Reutilización y uso de materiales reciclados.

18. Impacto de los materiales de construcción. Desmontaje, reutilización y reciclado al final del ciclo de vida.

19. Impactos generados en la fase de construcción. Residuos de construcción.

\section{Calidad del ambiente interior}

20. Toxicidad de los materiales de acabado interior.

21. Concentración de $\mathrm{CO}_{2}$ en el aire interior.

22. Limitación a la velocidad del aire en la zonas con ventilación mecánica.

23. Eficiencia de la ventilación en las áreas con ventilación natural.

24. Confort térmico en los espacios con ventilación natural.

25. Iluminación natural en los espacios de ocupación primaria.
26. Deslumbramiento en las zonas de ocupación no residencial.

27. Nivel de iluminación y calidad de la luz en los puestos de trabajo.

28. Protección frente al ruido a través de la envolvente y zonas de ocupación primaria.

29. Protección frente al ruido generado en el recinto de instalaciones en las zonas de ocupación primaria.

30. Protección frente al ruido entre aéreas de ocupación primaria. Particiones y medianeras.

\section{E Calidad del servicio}

31. Eficiencia de los espacios.

32. Disponibilidad de un sistema de gestión.

33. Capacidad de control local del sistema de iluminación, en las áreas de ocupación no residencial.

34. Capacidad de control local de los sistemas de calefacción, refrigeración y ventilación, en las áreas de ocupación no residencial.

35. Desarrollo e implementación de un plan de gestión de mantenimiento.

\section{F Aspectos sociales y económicos}

36. Mejora el acceso para personas con discapacidad.

37. Derecho al sol.

38. Acceso a espacios abiertos privados desde las viviendas.

39. Protección a las vistas del interior de las viviendas desde el exterior.

40. Acceso visual desde las áreas de trabajo.

41. Coste a lo largo del ciclo de vida. Coste de construcción.

42. Coste a lo largo del ciclo de vida. Coste de explotación.

Ante la necesidad de desarrollar un modelo aceptado por la comunidad científica sobre los impactos a evaluar y el método de cálculo asociado a los indicadores numéricos, ha sido de gran ayuda el trabajo desarrollado por los organismos de Normalización ISO (International Standards Organization) (4-6) y CEN(Comisión Europea de Normalización) (7-12).

Además, los indicadores incorporados contemplan aquellos que definen el perfil ambiental de España por sectores, recogidos en el sistema Español de indicadores $(13)^{10}$.

Las categorías de impacto establecidas en el modelo desarrollado se presentan en la Tabla 1. Para la cuantificación de dichos impactos 
Tabla 1

Propuesta de impactos e indicadores mínimos necesarios para la evaluación

\begin{tabular}{|c|c|c|}
\hline & IMPACTOS & Indicador \\
\hline 1 & Cambio climático & $\mathrm{kg} \mathrm{CO} \mathrm{CO}_{2}$ \\
\hline 2 & Aumento de las radiaciones UV a nivel del suelo & $\mathrm{kg} \mathrm{CFC}{ }_{11} \mathrm{eq}$ \\
\hline 3 & Pérdida de fertilidad & $\mathrm{kg} \mathrm{SO}_{2} \mathrm{eq}$ \\
\hline 4 & Pérdida de vida acuática & $\mathrm{kg} \mathrm{PO}_{4} \mathrm{eq}$ \\
\hline 5 & Producción de cáncer y otros problemas de salud & $\mathrm{kg} \mathrm{C}_{2} \mathrm{H}_{4} \mathrm{eq}$ \\
\hline 6 & Cambios en la Biodiversidad & $\%$ \\
\hline 7 & Agotamiento de energía no renovable, energía primaria & MJ \\
\hline 8 & Agotamiento de recursos no renovables diferentes de la energía primaria & $\mathrm{kg}$ de Sb \\
\hline 9 & Agotamiento de aguas potables & $m^{3}$ \\
\hline 10 & Uso del suelo & $m^{2}$ \\
\hline 11 & Agotamiento de suelo para depósito de residuos no peligrosos & $\mathrm{m}^{3}$ \\
\hline 12 & Peligro por la disposición o almacenamiento de residuos peligrosos & $\mathrm{kg}$ \\
\hline 13 & Peligro por la disposición o almacenamiento de residuos radiactivos & $\mathrm{kg}$ \\
\hline 16 & Salud, bienestar y productividad para los usuarios & $\%$ \\
\hline 19 & Riesgo financiero o de beneficios para los inversores - Coste del Ciclo de Vida & $€(E U R)$ \\
\hline
\end{tabular}

se requiere del uso de indicadores. En la Tabla 1 se presentan también los indicadores para el cálculo de los impactos.

El cálculo de la reducción de dichos impactos se realiza utilizando un método prestacional, que permite dar valores absolutos en la evaluación a partir de los indicadores, definidos como una variable que ha sido socialmente dotada de un significado añadido al derivado de su propia configuración científica, con el fin de reflejar de forma sintética una preocupación social con respecto al medio ambiente, e insertarla coherentemente en el proceso de toma de decisiones. El indicador ambiental se puede definir como una variable o estimación ambiental (por ejemplo, emisiones de $\mathrm{CO}_{2}$ ) que proporciona una información agregada y sintética sobre un fenómeno (por ejemplo, el cambio climático). Un indicador ambiental de un edificio debe señalar un aspecto medioambiental en términos de carga o impacto.

Para evitar confusión entre criterios e indicadores, es necesario interpretar los primeros como una propiedad física que debe ser medida y los segundos como una herramienta para medir esa propiedad física. Algunos indicadores pueden asociarse a un solo criterio; esto es, que pueden asociarse varios impactos para medir la misma propiedad física.

Sirvan como ejemplo algunos procedimientos de cálculo de algunos de los indicadores cuantitativos utilizados para la evaluación de impactos:

\section{Cambio climático}

$\mathrm{kg} \mathrm{CO}_{2} \mathrm{eq}=\mathrm{kg} \mathrm{CO}_{2}+\mathrm{kg} \mathrm{CO} \times 2+\mathrm{kg} \mathrm{N} \mathrm{N}_{2} \mathrm{O} 320+\mathrm{kg}$ $\mathrm{CH}_{4} \times 24,5$

Incremento de la radiación ultravioleta a nivel del suelo

kg CFC 11 eq = kg R22 y otros HCFCx0,05+kgHALON$1201 \times 1,4+\mathrm{kgHALON}-1202 \times 1,25+\mathrm{kgHALON}-$ $1211 \times 3+$ kgHALON-1301x10+

\section{Pérdida de fertilidad}

$\mathrm{kg} \mathrm{SO}_{2} \mathrm{eq}=\mathrm{kgSO}_{2}+\left(\mathrm{NO}_{2} * 0,7+\mathrm{NO} * 1,07+\mathrm{NH}_{3} * 1,88\right.$ $+\mathrm{HF}^{*} 1,6+\mathrm{HCL}^{*} 0,88$

Producción de cáncer y otros problemas de salud

$\mathrm{kg} \mathrm{C}_{2} \mathrm{H}_{4} \mathrm{eq}=\mathrm{kgC}_{2} \mathrm{H}_{4}+\mathrm{kgHALONx} 0,021+\mathrm{kgCH} 4$ $\mathrm{x} 0,007+\mathrm{kgCFC} / \mathrm{HCFCx} 0,021+\mathrm{kgNMVOCx0}, 4$

$\underline{\text { Pérdida de vida acuática }}$

$\mathrm{kg} \mathrm{PO}_{4}$ eq $=\mathrm{kgPO}_{4}+\mathrm{kg}$ DQOx2,02E-2 + kg Nitratos $\mathrm{x} 1,0 \mathrm{E}-1+$......

Toxicidad humana

kgtox $=\mathrm{kgNO}_{x} \times 0,78+\mathrm{kgSO}_{2} \times 1,2$ 
Agotamiento de recursos naturales (Abiotic Depletion Potential, ADP)

$$
\begin{aligned}
& A D P=\sum_{i} m_{i} \times A D P_{i}\left(Z_{i}\right)=\text { kgcarbón }^{*} \\
& 1,34 E^{-2}+\text { kggas* }^{*}, 87 E^{-2}+\text { kgpetróleo* }^{*}, 02 \\
& E^{-2}+\ldots
\end{aligned}
$$

\section{MODELO DE EVALUACIÓN}

El proceso de evaluación de la sostenibilidad de una edificación requiere definir una escala de rendimientos y puntuaciones para poder establecer criterios de ponderación de impactos.

El modelo desarrollado se estructura a partir de la evaluación de los criterios descritos anteriormente, calculando la reducción de impactos asociados a cada criterio a partir de valores de referencia y ponderados de acuerdo con las condiciones regionales.

La mayoría de los sistemas (LEED, BREEAM) presentan puntuación y pesos fijos desarroIlados para una región determinada, asociados a cada criterio. Estos sistemas ofrecen simplicidad y facilidad de aplicación al mercado edificatorio, como herramientas para realizar una evaluación comparativa; sin embargo esto no es posible cuando los edificios evaluados están localizados en diferentes regiones con condiciones diferentes, que son los casos más frecuentes.

El sistema que se presenta tiene una estructura que permite modificar explícitamente la importancia relativa de los parámetros que reflejen las diferencias en las prioridades en las distintas regiones, y así asegurar que el sistema produce resultados que son significativos para la localidad donde se ubica el edificio. Por ejemplo, la importancia del consumo de agua potable es ciertamente diferente en el sur de España que en Finlandia y, por tanto, el sistema debe contemplar esta diferencia.

\subsection{Estructura del sistema de evaluación}

La estructura del sistema de evaluación se establece como muestra la Figura 1. Los criterios de sostenibilidad se evalúan a partir de las medidas reductoras de impacto recogidas en las estrategias de diseño y sus factores de rendimiento, estando cada uno de ellos asociado con las cargas ambientales y a su vez asociado con las categorías de impacto. Cargas ambientales son el uso de recursos y la producción de residuos, olores, ruidos y emisiones nocivas para el suelo, agua y aire. Estas cargas ambientales están relacionadas con los impactos ambientales que pueden expresarse como categorías de impacto. Las categorías de impacto incluyen el agotamiento de recursos renovables y no renovables.

A cada criterio se le asocia una puntuación de referencia (benchmark). Estos valores se establecen a partir de la revisión de la reglamentación de la región, el análisis de los valores de rendimiento usuales del edificio en la zona, o por consenso entre un grupo de expertos.

En la herramienta VERDE, la puntuación se establece de 0 a 5 en la forma siguiente:

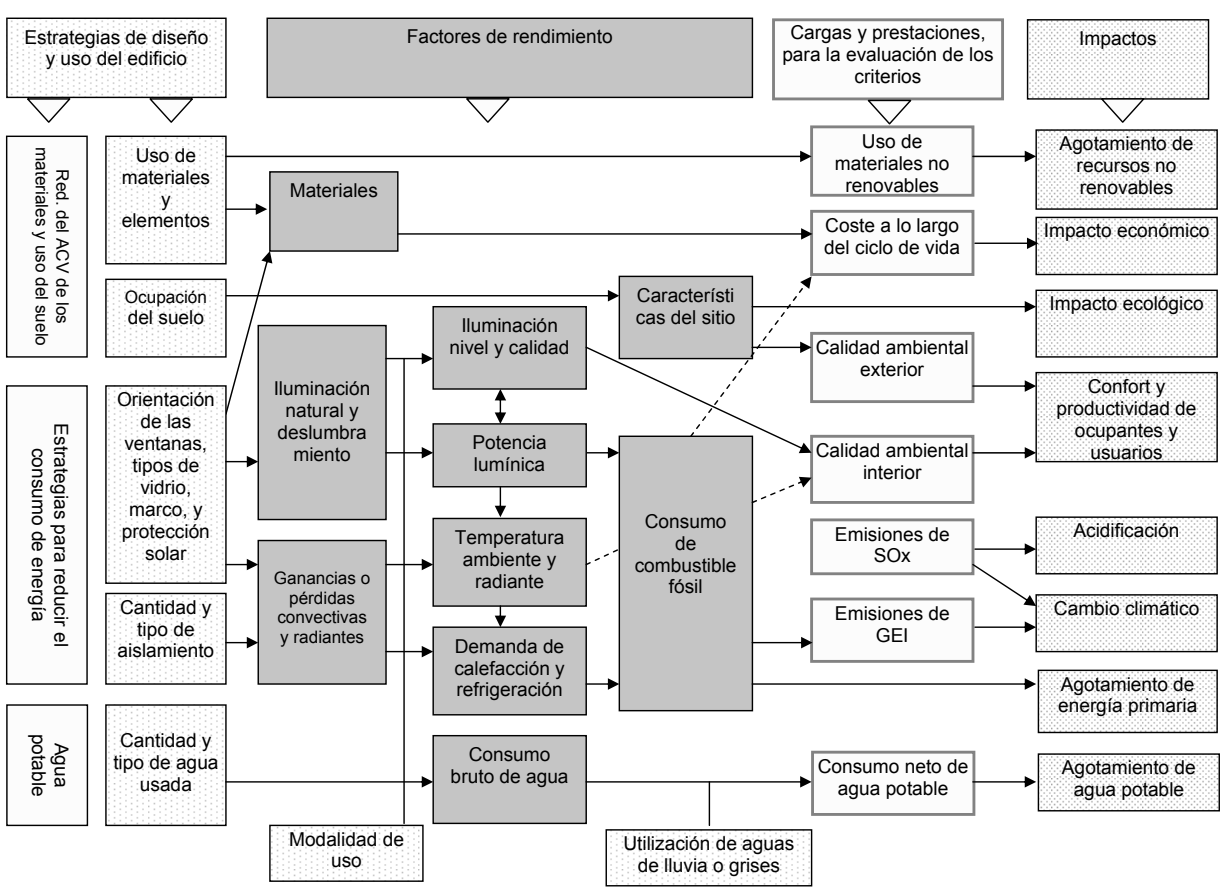


0 , valor de referencia que corresponde al cumplimiento normativo, práctica habitual o valor medio; 3, que define la calificación de una buena práctica; y 5 , que corresponde a la mejor práctica posible con un coste aceptable.

Los valores de rendimiento se estructuran de dos formas: orientados a datos, que describen los parámetros de rendimiento que pueden ser definidos mediante valores numéricos; y orientados a texto, que permiten describir varios niveles de rendimiento en forma de texto en aquellos criterios más subjetivos.

\subsection{Asignación de pesos al sistema de evaluación}

El sistema pesa cada uno de los impactos asociados a cada criterio según la extensión, la intensidad y la duración potencial de los efectos. La valoración de dichos criterios permite dar valores relativos en la evaluación.

El valor final de la evaluación se obtiene mediante la ponderación de los impactos reducidos en relación al edificio de referencia, cuya definición sigue la metodología prestacional. Los pesos asignados a cada impacto están relacionados con la importancia de dichos impactos en la situación mundial, en los impactos globales, y con la situación del entorno, en los impactos locales y regionales. Un análisis de la situación en España (14) nos lleva a justificar el peso asignado a las categorías de los impactos globales, cambio climático e incremento de la radiación UV a nivel del suelo, como sigue:

\section{Cambio climático (27\%)}

El cambio climático es quizás el principal problema ambiental de carácter global que tiene la humanidad actualmente, por ello el peso asignado a este impacto debe ser muy importante.

El $4^{\circ}$ informe de evaluación del International Panel of Climate Change - IPPC, presentado en París el 2 de febrero del año $2007^{11}$ pasará a la historia como el día en que desaparecieron las dudas acerca de si la actividad humana está provocando el cambio climático.

La temperatura de la Tierra subió el siglo pasado 0,76 grados, y subirá en los próximos cien años entre 1,8 y 4 grados, lo que tendrá efectos enormes en la biodiversidad, las Iluvias, las sequías, la subida del nivel del mar o la pérdida de los hielos polares. También influirá en los recursos alimenticios y en la sanidad, por citar sólo algunos de los impactos.

${ }^{11}$ www.marm.es

${ }^{12}$ www.aemet.es y para ello habrá que reducir las emisiones entre un $20 \%$ y un $30 \%$ hacia 2020 , y hasta un $80 \%$ en la segunda mitad del siglo XXI.

La concentración actual de gases de efecto invernadero es de 379 partes por millón (ppm) y aumenta a ritmo acelerado; en la era preindustrial (1650) era de 280 ppm. El aumento del $\mathrm{CO}_{2}$ se debe sobre todo al uso de combustibles fósiles y cambios en el uso de la tierra, y el del metano y del oxido nitroso, a la agricultura. Estos gases refuerzan el sistema invernadero natural de la Tierra, subiendo la temperatura.

Entre las posibles consecuencias para el planeta se pueden apuntar las siguientes:

Los científicos consideran que el 30\% de las especies del planeta estarán en grave riesgo de extinción si las temperaturas suben este siglo en torno a dos grados centígrados, lo que es muy probable como consecuencia del cambio climático. La salud, las infraestructuras, la disponibilidad de agua dulce o las cosechas en muchas regiones se verán también afectadas por el calentamiento, que tendrá globalmente efectos negativos si se supera ese nivel de temperaturas. Los impactos del cambio son ya visibles, y muestran el efecto de la actividad humana durante los últimos 30 años, aseguran los expertos.

El incremento de la temperatura ha tenido ya un fuerte efecto en el planeta: plantas que despiertan del invierno antes o mantienen las hojas más tiempo; especies de aves que han variado sus periodos de migración o cría; alteraciones de comunidades marinas debido al aumento de la temperatura del agua o cambios en la salinidad y las corrientes, entre otros.

\section{Y para España:}

Más inundaciones y tormentas, menos nieve, aguas más salinas, más sequías, más olas de calor y playas menos extensas. Se puede afirmar que el cambio climático afectará a España más que a otras regiones.

Este problema, "el mayor al que se enfrenta la humanidad" provocará que haya menos agua, sobre todo en el Mediterráneo, pero más lluvias torrenciales -con riesgo de inundaciones-. A finales de este siglo (2090-2099) las precipitaciones se habrán reducido en España un 20\% respecto al período 1980-1990.

Las temperaturas y las olas de calor seguirán aumentando. El año más caluroso de todos los que se tienen registros (desde 1850) ha sido 2006, según La Agencia Estatal de Meteorología $^{12}$. Los siguientes seis fueron 
posteriores a 1990. La temperatura media aumentó en España 0,9 grados centígrados en los 73 años que van de 1931 a 2004, mientras que la media mundial sólo subió 0,76 grados entre 1850 y 2005 (155 años).

Se calcula que podría haber una ola de calor cada tres o cinco años.

El cambio climático tendrá también efectos en las playas, que retrocederán 15 metros por la subida del nivel del mar antes de 2050. Ésta fue una de las conclusiones del informe "Impactos en la costa española por el efecto del cambio climático", presentado por el Ministerio de Medio Ambiente el pasado septiembre y elaborado por expertos de la Universidad de Cantabria ${ }^{13}$.

Adaptarse a esta nueva situación exigirá replantear el urbanismo de la costa y dejar de construir al borde del mar en lugares que se van a inundar. En algunas comunidades autónomas ya se prohíbe construir a menos de 500 metros del mar.

El aumento del nivel del mar no será homogéneo. Para el Cantábrico, el incremento previsto es de 35 centímetros, 20 en el Mediterráneo y unos 10 en el Golfo de Cádiz.

Las zonas más afectadas, por su poca altura, serán la Albufera de Valencia, Doñana (Huelva, Sevilla y Cádiz), la Costa Brava (Girona y Barcelona) y la Manga del Mar Menor (Murcia).

El nivel del mar sube por el deshielo de los glaciares, pero principalmente porque al calentarse, el agua ocupa más volumen.

Y el agua se calienta porque la quema de combustibles fósiles, como el carbón o el petróleo, produce gases de efecto invernadero que se acumulan en la atmósfera y dificultan la salida del calor que emite la Tierra.

Otros efectos del cambio climático serán la disminución de los recursos hídricos -entre un $5 \%$ y un $14 \%$ antes de 2030 -, cambios en la fecha de floración de plantas y en la reproducción de especies y un mayor riesgo de crecidas fluviales, tormentas e incendios forestales, según el informe Impactos del cambio climático en España presentado por el Ministerio de Medio Ambiente en 2005.

El clima árido, que actualmente sólo se da en Murcia y Almería, pasará a ocupar una mayor parte de Andalucía y parte de Castilla-La Mancha, según este estudio, en cuya elaboración participaron más de 400 científicos. El calentamiento tendrá una especial incidencia en dos sectores: el turismo y la agricultura.

\section{Incremento de la radiación UV a la altura del suelo $(0 \%)$}

El Perfil ambiental de España $2006^{14}$ (14) señala que las emisiones de gases que destruyen la capa de ozono se han reducido considerablemente como se muestra a continuación, por lo que el peso asociado a este impacto debe ser muy limitado. En el sector de la edificación, a falta de disponer de los datos sobre los impactos asociados a la fabricación de los materiales, el peso es nulo.

La evolución del consumo aparente (producción + importación - exportación) de los compuestos que agotan la capa de ozono (CFC, CCl4, halones, HCFC y metil-cloroformo) en España, expresado en toneladas ponderadas según el Potencial Agotador de la Capa de Ozono (PAO), ha disminuido de forma sustancial. Existe una tendencia clara a la reducción drástica en el consumo de estas sustancias, debido al calendario de eliminación de producción y consumo establecido por el Protocolo de Montreal de $1987^{15}$. En concreto, para los países desarrollados, se propuso el año 1994 para la eliminación total de la producción de halones, y el consumo aparente también se redujo sustancialmente en ese año, llegando a cero en 1996. Según el calendario del Protocolo de Montreal, la UE debía suprimir la elaboración de los CFC y el CCl4 para 1995 y el metil-cloroformo para 1996, lo que se ve reflejado en el consumo aparente, que en el año 1996 era cero para el $\mathrm{CCl} 4$ y el metil-cloroformo, y prácticamente ha desaparecido para los CFC.

El uso de los HCFC aumentó, como consecuencia de la puesta en marcha de esta regulación, como sustitutos de los CFC.

Sin embargo, aunque su efecto es sustancialmente menor, los HCFC también contienen cloro y afectan a la capa de ozono, por lo que su producción está regulada por el citado Protocolo, y se prevé que dejen de utilizarse en la Unión Europea para el año 2015. Están ya siendo sustituidos en muchas aplicaciones por HFC que, si bien no dañan la capa de ozono, son gases de efecto invernadero.

La asignación de un peso nulo a este impacto está basada en los datos aportados por el Observatorio de la Sostenibilidad en España y el Ministerio de Medio Ambiente en los que se observa que el estado del consumo aparente de sustancias que agotan la capa de ozono en España es nula desde el año 2000 (Figura 2, pág. 98).

De igual forma se ponderan los impactos regionales y locales, dependiendo del emplazamiento del edificio. Esto obliga a regionalizar las herramientas de evaluación

\footnotetext{
${ }^{13}$ Www.marm.es

${ }^{14}$ www.marm.es

${ }^{15}$ www.marm.es
} 
cuando se evalúen otros impactos además de los globales.

\subsection{Escala de análisis y sistema de puntuación}

Los edificios están formados por componentes y materiales, y existen en un contexto de infraestructura, área urbana e incluso extensos territorios. Aunque la mayor parte de los esfuerzos en la metodología propuesta se focalizan en edificios individuales, se pretende tener en consideración esas otras escalas de operación para permitir que el resultado del análisis a escala edificio pueda ser utilizado como entrada en un análisis posterior a escala superior, tal como un proyecto complejo de edificios o incluso un barrio.

El sistema de puntuación convierte los valores dimensionales de los indicadores en una puntuación final acerca del rendimiento global del edificio. La puntuación final se asigna a un intervalo [0; Smax], donde Smax es la máxima puntuación que puede ser alcanzada, correspondiente al mejor rendimiento global. Su cómputo se realiza en dos pasos: normalización de los valores de los indicadores, asociando una puntuación al valor de cada indicador, y agrupación de las puntuaciones para producir una puntuación final.

La función de normalización convierte el valor de los indicadores (por ejemplo 50 $\mathrm{kWh} / \mathrm{m}^{2}$ año de consumo de energía no renovable) en una puntuación adimensional normalizada en un intervalo específico (por ejemplo de 0 a 5 en la herramienta VERDE). Cada valor del indicador puede ser normalizado de diferente manera (lineal o no lineal), dependiendo de sus características.

Los rangos traducen los valores de un determinado indicador de los sujetos de valoración en una puntuación concreta, cuya principal característica es su operatividad con los obtenidos en otros indicadores para dar una valoración final. A su vez, informan de la posición del valor obtenido dentro del universo de sujetos que la herramienta reconoce, con lo que se aporta una valoración relativa que aporta datos sobre el edificio respecto a sus semejantes.

Los rangos pueden distribuir la puntuación desde una función continua (valor del indicador) hacia otra función continua (puntuación obtenida), con lo que se admiten puntuaciones fraccionarias; desde una función continua hasta unos valores discretos, en los que la puntuación admite sólo valores enteros y se hace sobre unos entornos cerrados definidos como valores del indicador; desde unos valores discretos de indicador (por ejemplo cuando se juzgan acciones), hasta unos valores discretos de puntuación.

Valor de referencia, es aquel valor exigido por la normativa; si no existe normativa, el que corresponde a las buenas prácticas o el valor medio extraído de la estadística.

En VERDE, el valor 0 debe corresponder al valor del edificio de referencia que actúa como contraste frente al edificio que se está valorando. Las medidas deben servir para reducir el impacto ambiental, y eso debe hacerse sobre una referencia que no puede ser otra que la realidad actual expresada de la mejor forma posible.

Valor máximo. Es aquel valor que se obtiene aplicando las mejores técnicas disponibles, económicamente viables.

La máxima puntuación debería ser, teóricamente, el valor sostenible: esa es la imagen que se quiere alcanzar y, por tanto, el referente de máxima puntuación. Eso implica disponer de ese referente de edificio sostenible. Hoy parece razonable que, aunque se indique como referencia el valor correspondiente a la sostenibilidad, el valor máximo del indicador al que le va a corresponder la puntuación máxima sea el que pueda obtenerse en la edificación actual, tomando las mejores opciones disponibles, basadas en soluciones técnicamente solventes, económicamente viables y socialmente aceptables.

Interpolación. La función que relaciona los valores del indicador con la puntuación que le corresponde es la que establece realmente la relación entre el indicador y el impacto ambiental (o la calidad) que genera. Existe pues, y sin ello es difícilmente justificable la presencia del indicador en el sistema, una función que relaciona los valores del indicador con magnitudes de impacto ambiental en el ámbito que sea.

En el más sencillo de los casos, cuando los valores del indicador y las puntuaciones de los rangos son magnitudes continuas, la línea recta es la función que relaciona un indicador que tiene una relación (lineal) inmediata con el impacto.

En otros casos, curvas logarítmicas o de otro tipo son las apropiadas. En VERDE, entre el valor de referencia (0) y el máximo obtenible (5), se realiza una interpolación lineal.

Cuando los valores del indicador o los de la puntuación son discretos, no hay función sino una estimación sobre situaciones concretas que finalmente determina una lista de comprobación (check-list). 


\subsection{Los materiales de construcción y el edificio}

Los edificios se construyen y mantienen mediante el uso de un gran número de materiales y productos de construcción, durante diferentes fases de su ciclo de vida. En la evaluación del rendimiento ambiental del edificio juegan un importante papel los materiales, por lo que el conocimiento de sus características y los impactos ambientales asociados a la producción, uso y mantenimiento juegan un importante papel en la evaluación global del edificio.

Los organismos de Normalización ISO y CEN están trabajando al respecto en varios proyectos normativos para cubrir estos aspectos, referidos en concreto a las declaraciones ambientales de producto ( $E n-$ vironmental Product Declaration, EPD) (6), las reglas de categorización de productos (Product Category Rules, PCR) (12) y los formatos de comunicación. Sin embargo, hasta que se pueda disponer en España con facilidad de la declaración medioambiental de productos de la construcción pasarán todavía unos años, por lo que la herramienta VERDE evalúa de momento tan solo los impactos, la energía incorporada a los materiales y las emisiones de $\mathrm{CO}_{2}$, en la mayor parte de los casos, utilizando datos de materiales genéricos.

\section{LA HERRAMIENTA DE EVALUACIÓN VERDE}

\subsection{Descripción}

La herramienta sigue la misma metodología que la Certificación Energética de Edificios en la opción prestacional; calcula la reducción de impacto del edificio objeto, comparado con un edificio de referencia, siendo definido como tal el mismo edificio que cumple las exigencias mínimas fijadas por la normativa y reglamentos, en aquellos criterios a los que se aplica dicha normativa, y que sigue la práctica habitual, en aquéllos a los que no se aplica. La herramienta evalúa criterios asociados a los impactos que produce un edificio a lo largo de todo su ciclo de vida, y se implementa la evaluación de los impactos asociados a cada criterio definidos en la normativa ISO y CEN.

También se utilizan los resultados de la certificación energética del edificio como dato para la evaluación de los criterios agrupados en la categoría "Energía y atmósfera".

De este modo, VERDE analiza un total de 42 criterios, repartidos según la tipología de los edificios a evaluar.
La herramienta, en su versión 1.0, se estructura en dos programas (hojas de cálculo):

Hoja A.- Destinada al equipo que mantiene los datos, determina los criterios obligatorios a analizar, modifica las valoraciones por modificación de exigencias normativas como Decretos de las Comunidades Autonómicas, Ordenanzas Municipales, etc.

Hoja B.- Destinada al usuario, en ella se introducen los datos del edificio, los resultados del cumplimiento normativo (Ordenanzas, clase energética...), selecciona los criterios aplicados a su proyecto (además de los obligatorios) y las puntuaciones asociadas a cada criterio y presenta los resultados de la evaluación.

Los criterios evaluados en VERDE - uso residencial, y los objetivos a alcanzar son los siguientes:

0. Vida útil del edificio.

Este criterio valora y premia la consecución de periodos de vida útil garantizados superiores a los mínimos reglamentarios establecidos para las diferentes tipologías de estructuras, con la finalidad de amortizar los impactos iniciales producidos por la ejecución del edificio.

1. Estrategias para la clasificación y el reciclaje de residuos.

Los objetivos del criterio son, promover y premiar la existencia de locales en el interior o exterior del edificio para la separación, almacenamiento temporal y reciclaje de residuos domésticos.

Asegurar que los residuos sólidos, orgánicos e inorgánicos sean recogidos, separados y reciclados. Animar una cultura del reutilizar y reciclar.

Utilizar los residuos vegetales para generación de compost y el abonado de zonas verdes. Los residuos orgánicos se reciclan en plantas de biogás para la generación de energía eléctrica, gracias al gas generado en el proceso de digestión.

Separar los residuos de plásticos, cartones-papeles, vidrios, pilas y otros, para su traslado a plantas de reciclado.

2. Uso de plantas autóctonas.

Este criterio premia la promoción y el uso de plantas autóctonas en los espacios verdes. 
3. Uso de árboles para crear áreas de sombra.

Disminuir el efecto de la radiación sobre las fachadas del edificio, para la reducción de la demanda de refrigeración en verano, sin que afecte el soleamiento en invierno.

4. Efecto isla de calor a la altura del suelo.

Disminuir el efecto de isla de calor en áreas urbanas, mediante la utilización de materiales de alta reflectancia o de zonas verdes en los espacios exteriores.

5. Efecto isla de calor a la altura de la cubierta.

Disminuir el efecto de isla de calor en áreas urbanas, mediante la utilización de materiales de alta reflectancia o de zonas verdes en las cubiertas.

6. Contaminación lumínica.

Reducir las pérdidas de energía eléctrica utilizada para la iluminación de los espacios exteriores de la parcela, evitando el derroche de energía que se emite fuera de un cono de $120^{\circ}$ por la luminaria.

7. Uso de energía no renovable incorporada en los materiales de construcción.

Reducir los impactos asociados al consumo de energía no renovable incorporada en los materiales de construcción, mediante la elección de materiales con bajo consumo de la misma durante su proceso de extracción y transformación así como mediante el uso de materiales reutilizados y/o reciclados.

8. Energía no renovable en el transporte de los materiales de construcción.

Reducir la cantidad de energía no renovable utilizada en el transporte de los materiales de construcción, incentivando el uso de materiales locales.

9. Consumo de energía no renovable durante la fase de uso. Demanda y eficiencia de los sistemas.

Promover y premiar la reducción del consumo de energía no renovable necesaria para la climatización del edificio (calefacción y refrigeración) y ACS en los edificios del sector residencial.
Reducir la cantidad de energía no renovable consumida por el uso del edificio, aplicando medidas pasivas de diseño para la reducción de la demanda energética y la eficiencia de los sistemas.

10. Demanda de energía eléctrica en la fase de uso.

Promover y premiar la reducción del consumo de energía no renovable necesaria para la iluminación y electrodomésticos en los edificios del sector residencial.

El criterio valora el ahorro de energía estimado por el uso de sistemas y equipos eficientes para la iluminación y otros equipos eléctricos consumidores de energía, diferentes de los consumos para calefacción, refrigeración y ACS.

11. Producción de energía renovable en la parcela.

Promover y premiar la reducción del consumo de energía no renovable, a partir de la instalación de sistemas que permitan la generación de energía mediante fuentes renovables.

El modo de conseguir los objetivos de este criterio pasa por la integración en el edificio o parcela de sistemas de producción de energía a través de fuentes renovables que excedan las exigencias mínimas establecidas por el CTE.

12. Emisiones de sustancias foto-oxidantes en procesos de combustión.

Promover y premiar la reducción de emisiones de productos foto-oxidantes precursores de la creación de ozono troposférico.

El modo de conseguir los objetivos de este criterio pasa por la instalación de calderas que generen baja emisión de NOx en la fase de uso del edificio.

13. Emisiones de sustancias que reducen el ozono estratosférico.

Promover y premiar la reducción del uso de productos que destruyen la capa de ozono, equipos que los incorporen o su manipulación durante la fase de explotación del edificio. 
El impacto asociado a las emisiones de estos gases, debido a los materiales de construcción, es evaluado en el criterio Impacto de los materiales de construcción.

14. Consumo de agua potable.

Reducir el consumo de agua potable en la fase de uso del edificio, mediante medidas de ahorro y eficiencia.

15. Retención de aguas de lluvia para su reutilización.

Promover un sistema de gestión de aguas superficiales, de recogida y almacenamiento de las aguas de lluvia para su reutilización.

16. Reutilización de aguas grises.

Reducir el consumo de agua potable para el uso del edificio, mediante la instalación de un sistema de recuperación y reutilización de las aguas grises.

17. Impacto de los materiales de construcción. Reutilización y uso de materiales reciclados

Reducir los impactos asociados a la producción de los materiales de construcción, mediante la elección de materiales con bajos impactos durante su proceso de extracción y transformación, así como mediante el uso de materiales reutilizados y/o reciclados.

18. Impacto de los materiales de construcción. Desmontaje, reutilización y reciclado al final del ciclo de vida.

Promover un diseño que pueda facilitar el desensamblaje de sus componentes, a fin de que puedan ser reutilizados o reciclados al final de la vida útil del edificio.

19. Impacto generados en la fase de construcción. Residuos de construcción.

Reducir los residuos generados durante la obra del edificio, con el uso de elementos prefabricados e industriales, o empleando procesos de obra controlados que minimicen la producción de residuos.

20. Toxicidad de los materiales de acabado interior.

Promover y premiar el uso de materiales de acabado que no pongan en riesgo la salud de los ocupantes, y la eliminación, previa la ocupación, de los contaminantes emitidos por los materiales de terminación interior, para reducir los problemas de calidad del aire interior del edificio resultantes del proceso de construcción.

21. Eficiencia de la ventilación en las áreas con ventilación natural.

Premiar la existencia de condiciones que promuevan la ventilación natural, de manera que no sea necesario recurrir a otras formas de ventilación para garantizar un nivel de renovación del aire interior que salvaguarde su calidad y reduzca la exposición de los ocupantes a contaminantes interiores nocivos para la salud.

Asegurar que el número, colocación y tipo de ventanas $u$ otras aberturas en un edificio con ventilación natural, sea capaz de garantizar un nivel alto de calidad de aire y ventilación.

22. Confort térmico en los espacios con ventilación natural.

Promover y premiar el control de temperatura interior dentro de los rangos establecidos por zona climática, a través de la ventilación natural.

23. Iluminación natural en los espacios de ocupación primaria.

Promover y premiar un nivel adecuado de iluminación natural durante el día en todos los espacios de ocupación primaria.

24. Protección frente al ruido a través de la envolvente y zonas de ocupación primaria.

Promover y premiar el aislamiento acústico de la evolvente entre el exterior y los recintos protegidos.

25. Protección frente al ruido generado en el recinto de instalaciones en las zonas de ocupación primaria.

Promover y premiar el aislamiento acústico frente a ruido aéreo y de impacto entre los recintos de instalaciones y los recintos protegidos.

26. Protección frente al ruido entre áreas de ocupación primaria. Particiones y medianeras.

Promover y premiar el aislamiento acústico entre recintos protegidos y recintos 


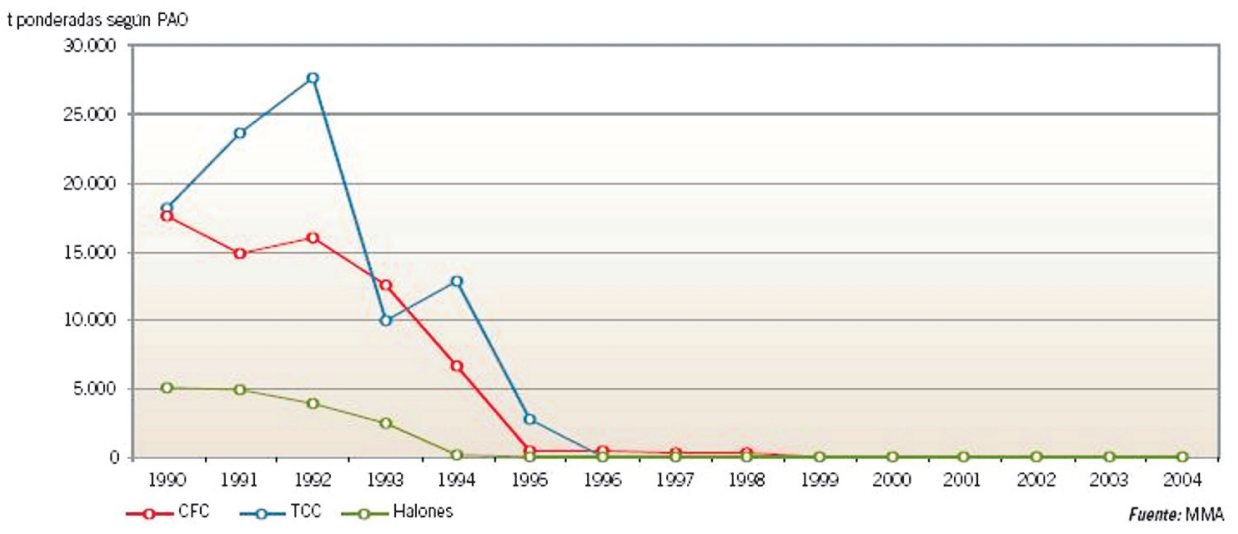

pertenecientes a otras unidades de uso o de la misma unidad de uso.

27. Eficiencia de los espacios.

Incentivar un diseño que distribuya el espacio de forma eficiente y funcional, aprovechando la superficie disponible para zonas de ocupación y usos primarios y reduciendo la superficie empleada en elementos de construcción y/o elementos de distribución o que no respondan al uso del edificio.

28. Desarrollo e implementación de un plan de gestión de mantenimiento.

Promover la elaboración de un plan de mantenimiento del edificio detallado, completo e inteligible por los usuarios finales, que sea extensible a toda la vida útil del edificio.

29. Mejora del acceso para personas con discapacidad.

Permitir o mejorar el acceso y uso de los servicios y equipamientos para personas con discapacidad física.

30. Derecho al sol.

Promover un diseño sostenible que asegure un soleamiento directo a las áreas habitadas principales de las viviendas durante las horas centrales del día de solsticio de invierno.

31. Acceso a espacios abiertos privados desde las viviendas.

Incentivar la creación de espacios abiertos privados en las viviendas (terrazas, patios, etc.).
32. Protección a las vistas del interior de las viviendas desde el exterior.

Promover un diseño sostenible que asegure la intimidad en el interior de las viviendas.

33. Coste a lo largo del ciclo de vida. Coste de construcción.

Promover un diseño sostenible que no implique un incremento en el coste de construcción sobre el de un edificio convencional.

34. Coste a lo largo del ciclo de vida. Coste de explotación.

Promover un diseño sostenible que suponga una reducción del coste, durante la fase de explotación del edificio, en los consumos cuantificables del mismo.

El sistema se focaliza en la evaluación de los impactos durante la construcción, uso y fin de la vida, es decir en las diferentes fases del ciclo de vida del edificio. Sin embargo, los datos referidos a la fase de uso son los más significativos.

En relación a los datos de los materiales se hace referencia a las declaraciones ambientales de producto, a pesar de que actualmente existe solo para un restringido número de productos y la información se limita a la energía incorporada y a las emisiones de $\mathrm{CO}_{2}$.

La evaluación de las prestaciones se especifica en las referencias (benchmark), asociando una puntuación que va de 0 , práctica común, a un máximo de +5 como mejor práctica posible. En la Tabla 2, correspondiente al 
criterio "Uso de plantas autóctonas", se muestra una valoración de tipo cuantitativo.

En la Tabla 3, correspondiente al criterio "Clasificación y reciclaje de residuos sólidos urbanos", se muestra una evaluación de tipo cualitativo: en un texto se describen de forma cualitativa los tres niveles de prestación asociados a la puntuación del criterio. En estos casos las fichas para la valoración del criterio son como las del ejemplo que se presenta a continuación.

\subsection{Resultados}

Existen dos resultados que responden a dos tipos de cálculo diferentes: Resultados de la evaluación relativa, y resultados de la evaluación absoluta.

\section{Relativa:}

Responde al cálculo del porcentaje de la reducción de cada uno de los impactos evaluados, respecto del edificio de referencia, a partir de la implantación de medidas de sostenibilidad evaluadas en cada criterio (Figura 3).

Absoluta: Responde al cálculo de reducción de impactos reales, medidos en las unidades asociadas a cada tipo de impacto (por ejemplo, el cambio climático, en $\mathrm{kg}$ de $\mathrm{CO}_{2}$ equivalente), Figura 4. Se mide respecto a un edificio de referencia que representa la norma vigente y la práctica habitual de la región (valor 0 ), y toma como máximo la mejor práctica posible (valor 5). El resultado final para comparar edificios se obtiene ponderando los tipos de impacto que se van a evaluar y el peso que ha sido asignado a cada uno de ellos. El peso asignado sirve para ponderar los resultados parciales obtenidos en cada área de impacto y para formular un valor final, que se expresa en
Tabla 2

Ejemplo de puntuación del criterio cuantitativo. A2.

\begin{tabular}{|l|l|c|}
\hline A 2 & Uso de plantas autóctonas \\
\hline Método de evaluación & $\begin{array}{l}\text { La evaluación del edificio a través de este criterio se establece por medio del Porcentaje de } \\
\text { Áreas Verdes Ocupadas por Plantas Autóctonas }\left(\mathrm{P}_{\text {AuT }}\right) \text { como resultado del cociente entre el } \\
\text { área verde de plantas autóctonas y el área libre total. }\end{array}$ \\
\hline PUNTUACIÓN (BENCHMARK) & $\begin{array}{l}\text { Ratio entre área verde de plantas autóctonas y la superficie } \\
\text { ajardinada en la parcela }\end{array}$ & $30 \%$ \\
\hline Práctica habitual & & $72 \%$ \\
\hline Buena práctica & & $100 \%$ \\
\hline Mejor práctica &
\end{tabular}

Tabla 3

Ejemplo de puntuación del criterio cualitativo. A1.

\begin{tabular}{|l|l|l|}
\hline A 1 & \multicolumn{2}{|c|}{ Estrategias para la clasificación y el reciclaje de residuos en el edificio } \\
\hline Medidas & \multicolumn{1}{|c|}{ Descripción } & Valoración \\
\hline 1.1 & $\begin{array}{l}\text { Se prevé la recogida y transporte hasta un punto de recogida municipal de todas } \\
\text { aquellas fracciones de residuos que no tengan una recogida selectiva pública en la } \\
\text { puerta del edificio. Normalmente se recogen en la puerta del edificio las secciones de } \\
\text { envases y otros. }\end{array}$ & $P_{\mathrm{RSU}}=31 \%$ \\
\hline 1.2 & $\begin{array}{l}\text { Se prevé un lugar donde almacenar muebles y enseres y otros residuos NO } \\
\text { peligrosos que se generen de forma puntual durante el uso del edificio hasta su } \\
\text { recogida por los servicios públicos o su traslado hasta un lugar donde se produzca } \\
\text { dicha recogida. }\end{array}$ & $P_{\mathrm{RSU}}=19 \%$ \\
\hline 1.3 & $\begin{array}{l}\text { Se proyecta algún sistema que permita reciclar los residuos orgánicos generados } \\
\text { dentro de la propia parcela, por ejemplo, compostaje de residuos orgánicos para } \\
\text { abonar las zonas verdes del edificio o la parcela. }\end{array}$ & $P_{\mathrm{RSU}}=40 \%$ \\
\hline
\end{tabular}

ॠ $\%$ Reducción de Impacto $\quad \square \%$ Impacto Residual

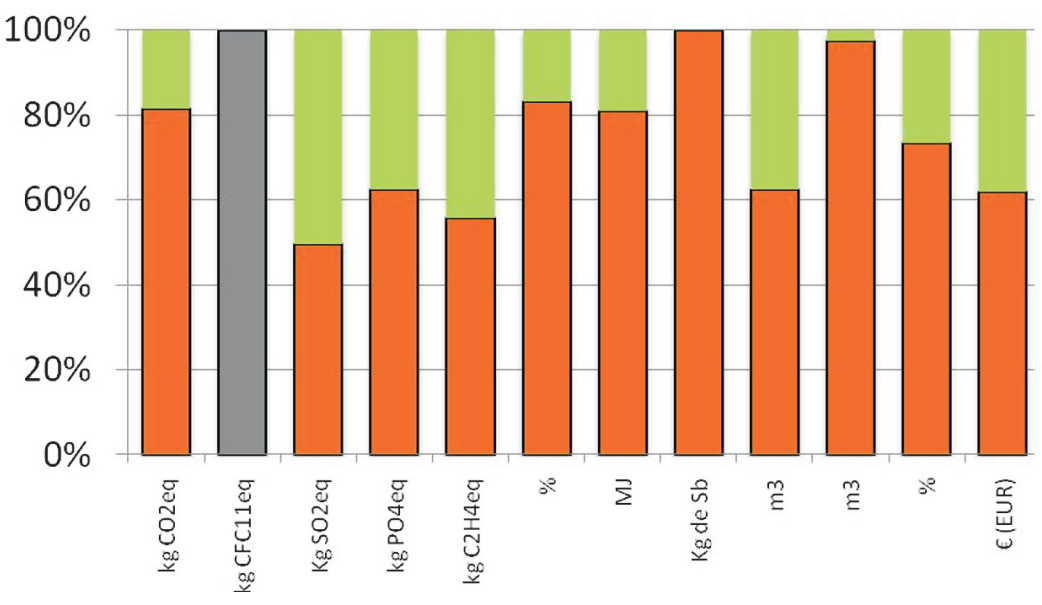

\begin{tabular}{|c|c|c|c|c|c|c|c|c|c|}
\hline \multicolumn{10}{|c|}{ Resultados de la evaluación absoluła } \\
\hline$\#$ & $\begin{array}{l}\text { Los datos estan basados en las puntuaciones } \\
\text { obtenidas en la autoevaluación }\end{array}$ & $\begin{array}{l}\text { Indicador/ } \mathbf{m} 2 \\
\text { año }\end{array}$ & Pesos & $\begin{array}{l}\text { Ediliclo de } \\
\text { referencia }\end{array}$ & Edificio evaluado & Impacto evitado & $\begin{array}{l}\text { \% de ereducín de } \\
\text { impacto }\end{array}$ & \% de impacto & $\begin{array}{c}\text { Impocto relativo } \\
\text { eviltado }\end{array}$ \\
\hline 1 & Cambio climático & $\mathrm{kg} \mathrm{CO} 2 \mathrm{eq}$ & $27 \%$ & 56,11 & 32,90 & 23,21 & $41,4 \%$ & $58.6 \%$ & 2,1 \\
\hline 2 & Aumento de las radiaciones UV a nivel del suelo & $\mathrm{kgCFClleq}$ & $2 \%$ & 0.00 & 0,00 & 0.00 & $0.0 \%$ & $100.0 \%$ & 0.0 \\
\hline 3 & Pérdida de fertilidad & $\mathrm{Kg} \mathrm{SO} 2 \mathrm{eq}$ & $5 \%$ & 2,68 & 1,50 & 1,17 & $44,0 \%$ & $56,0 \%$ & 2,2 \\
\hline 4 & Pérdida de vida acuática & $\mathrm{kg} \mathrm{PO} 4 \mathrm{eq}$ & $6 \%$ & 0,14 & 0,11 & 0,03 & $21,4 \%$ & $78,6 \%$ & 1,1 \\
\hline 5 & Producción de cáncer y otros problemas de salud & $\mathrm{kg} \mathrm{C} 2 \mathrm{H} 4 \mathrm{eq}$ & $8 \%$ & 0.13 & 0.09 & 0.04 & $30,8 \%$ & $69,2 \%$ & 1.5 \\
\hline 6 & Cambios en la Biodiversidad & $\%$ & $4 \%$ & 700,00 & 300,00 & 400.00 & $57,1 \%$ & $42,9 \%$ & 2.9 \\
\hline 7 & $\begin{array}{l}\text { Agotamiento de energia no renovable, energía } \\
\text { primaria }\end{array}$ & MJ & $8 \%$ & 520,38 & 382,81 & 137,57 & $26.4 \%$ & $73.6 \%$ & 1,3 \\
\hline 8 & $\begin{array}{l}\text { Agotamiento de recursos no renovables diferentes de } \\
\text { la energia primaria }\end{array}$ & $\mathrm{Kg}$ de Sb & $9 \%$ & 102,25 & 98,54 & 3.71 & $3.6 \%$ & $96.4 \%$ & 0.2 \\
\hline 9 & Agotamiento de aguas potables & m3 & $10 \%$ & 3.73 & 1.54 & 2,19 & $58.8 \%$ & $41,2 \%$ & 2,9 \\
\hline 11 & Generación de residuos no peligrosos & m3 & $4 \%$ & 57,25 & 45,08 & 12,17 & $21,3 \%$ & $78,7 \%$ & 1.1 \\
\hline 16 & Salud, bienestar y productividad para los usuarios & $\%$ & $12 \%$ & 1.00 & 0.61 & 0.39 & $38,6 \%$ & $61,4 \%$ & 1.9 \\
\hline 19 & $\begin{array}{l}\text { Riesgo financiero o de beneficios para los inversores - } \\
\text { Coste del Ciclo de Vida }\end{array}$ & $€($ EUR) & $5 \%$ & 46,22 & 21,22 & 25,00 & $54,1 \%$ & $45,9 \%$ & 2,7 \\
\hline & Impacto evitado & & $100 \%$ & & & & & & 1,80 \\
\hline
\end{tabular}

3. Resultados de la valoración de la reducción de impactos por la aplicación de medidas sostenibles (en gris el impacto radiación ultravioleta a nivel del suelo cuyo peso en los criterios evaluados es nulo).

4. Resultados absolutos de la reducción de impactos. 
5. Resultado final de la evaluación.

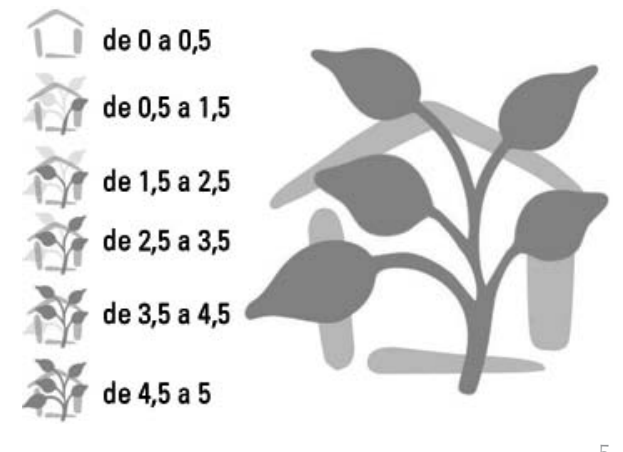

una puntuación que va de 0 a 5, Hoja Impactos (Impacts).

El resultado final se expresa según el número de "hojas sostenibles", con la correspondiente puntuación (Figura 5).

\section{CONCLUSIONES}

La metodología y herramienta presentada, "VERDE", permite evaluar la sostenibilidad de un edificio y su entorno, utilizando la técnica de adicción de impactos de una forma objetiva. La regionalización de la herramienta permite asignar pesos a los impactos regionales y locales, frente a otras herramientas de evaluación que no permiten dicha asignación local. La metodología presentada recoge los últimos desarrollos normativos y los incorpora para definir los valores de referencia en cada uno de los criterios analizados, las categorías de impactos a analizar y los métodos de cálculo de los indicadores para la valoración de los impactos.

La ponderación de los impactos sólo es exigible si se quiere proporcionar un resultado numérico global de la evaluación, ya que los valores de la reducción de cada uno de los impactos analizados nos permite dar un diagnóstico de la sostenibilidad del edificio y actuar en aquellas medidas asociadas para la reducción de dichos impactos.

Esta metodología permite exportar el sistema a cualquier región del planeta sin más que definir el número de criterios e impactos a analizar seleccionados de una base de datos general y modificar los valores de referencia y máximos alcanzables en cada uno de criterios e impactos seleccionados.

\section{BIBLIOGRAFÍA}

(1) Thomas Saunders. A discussion document comparing International environmental assessment methods for buildings. BRE, March 2008.

(2) Zhuguo Li. A new life cycle impact assessment approach for buildings. Building and Environment, 41 (2006) 1414-1422.

(3) W.L. Lee, F.W.H. Yik, J. Burnett. Assessing energy performance in the latest versions of Hong Kong Building Environmental Assessment Method (HK-BEAM). Energy and Buildings, 39 (2007) 343-354.

(4) ISO/FDIS 15392 Sustainability in building construction - General Principles.

(5) ISO/TS 21929 Sustainability in building construction - Sustainability indicators- Part 1: Framework for the development of indicators for buildings.

(6) ISO 21930 Sustainability in building construction - Environmental declaration of building products.

(7) prEN 15643-1 Sustainability of construction works - Integrated assessment of building performance. Part 1: General framework.

(8) prEN 15643-2 Sustainability of construction works - Assessment of buildings - Part 2: Framework for the assessment of the environmental performance.

(9) prEN 15643-3 Sustainability of construction works - Integrated assessment of building performance. Part 3: Framework for the assessment of social performance.

(10) prEN 15643-4 Sustainability of construction works - Integrated assessment of building performance. Part 4: Framework for the assessment of economic performance.

(11) WI 3500011 Sustainability of construction works - Assessment of environmental performance of buildings - Calculation methods.

(12) prEN 15804 Sustainability of construction works - Environmental product declarations - Product Category Rules.

(13) Indicadores ambientales. Una propuesta para España, Ministerio de Medioambiente, Madrid, 1996.

(14) Perfil ambiental de España 2006, Ministerio de Medioambiente, 2007. 